\title{
Development of a TaqMan loop-mediated isothermal amplification assay for the rapid detection of pigeon paramyxovirus type 1
}

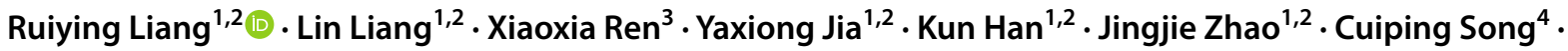 \\ Shangjin Cui ${ }^{1,2}$
}

Received: 12 August 2020 / Accepted: 2 December 2020 / Published online: 23 March 2021

(c) The Author(s), under exclusive licence to Springer-Verlag GmbH, AT part of Springer Nature 2021

\begin{abstract}
Pigeon paramyxovirus-1 (PPMV-1) is a strain of Newcastle disease virus (NDV) that has adapted to infect pigeons and poses a constant threat to the commercial poultry industry. Early detection via rapid and sensitive methods, along with timely preventative and mitigating actions, is important for reducing the spread of PPMV-1. Here, we report the development of a TaqMan loop-mediated isothermal amplification assay (TaqMan-LAMP) for rapid and specific detection of PPMV-1 based on the $F$ gene. This system makes use of six novel primers and a TaqMan probe that targets nine distinct regions of the $F$ gene that are highly conserved among PPMV-1 isolates. The results showed that the limit of detection was 10 copies $\mu \mathrm{L}^{-1}$ for PPMV-1 cDNA and $0.1 \mathrm{ng}$ for PPMV-1 RNA. The reaction was completed within 25 min and was thus faster than conventional RT-PCR. Moreover, no cross-reactions with similar viruses or with peste des petits ruminants virus (PPRV) or NDV LaSota vaccine strains were observed under the same conditions. To evaluate the applicability of the assay, the TaqMan-LAMP assay and a commercial RT-PCR assay were compared using 108 clinical samples, and the concordance rate between two methods was found to be $96.3 \%$. The newly developed PPMV-1 TaqMan-LAMP assay can therefore be used for simple, efficient, rapid, specific, and sensitive diagnosis of PPMV-1 infections.
\end{abstract}

\section{Introduction}

Newcastle disease virus (NDV), also referred to as avian paramyxovirus 1 (APMV-1), is highly infectious and has devastating effects on commercial poultry and other bird species [2, 11, 22]. The broad circulation of NDV in poultry has led to genetic divergence of virus strains into two groups,

Handling Editor: Bert K. Rima.

Cuiping Song

songcuiping@cahec.cn

Shangjin Cui

cuishangjin@caas.cn

1 Institute of Animal Science, Chinese Academy of Agricultural Sciences, Beijing 100193, China

2 Beijing Scientific Observation and Experimental Station of Veterinary Drugs and Diagnostic Technology, Ministry of Agriculture, Beijing 100193, China

3 China Institute of Veterinary Drug Control, Beijing 100081, China

4 China Animal Health and Epidemiology Center, Qingdao 266032, China referred to as class I and class II. Class I includes three subgenotypes, class II contains at least 20 identified genotypes (from I to XXI without XV), and pigeon paramyxovirus 1 (PPMV-1) belongs to class II, genotype VI (VI.1.1) [1]. PPMV-1 can be distinguished as unique antigenic variant of NDV by the hemagglutination inhibition (HI) assay [5].A member of the family Paramyxoviridae, subfamily Paramyxovirinae, and genus Avulavirus, PPMV-1 has a singlestranded, negative-sense RNA genome that is approximately $15.5 \mathrm{~kb}$ in length. This RNA contains six genes encoding the following six structural proteins (from 5' to 3'): RNAdependent RNA polymerase (L), hemagglutinin-neuraminidase $(\mathrm{HN})$, fusion $(\mathrm{F})$, matrix $(\mathrm{M})$, phosphoprotein $(\mathrm{P})$, and nucleoprotein (NP) $[7,26]$. In addition, $\mathrm{W}$ and $\mathrm{V}$ proteins are produced by RNA editing and are generated during transcription of the $P$ gene [25]. The cleavage site of the $\mathrm{F}$ protein is ${ }^{112} \mathrm{G} / \mathrm{RRQKRF}^{117}$ [9], and this feature is generally characteristic of PPMV-1. PPMV-1 was first reported in Italy in 1981 [3] and subsequently reached epidemic levels, spreading via racing and show pigeons to all regions of the world [24]. In China, PPMV-1 was first reported in 1985 in exotic pigeons, and it subsequently spread throughout the country $[11,26]$. The mortality rate in pigeons is $70 \%[10$, 
13, 24]. Therefore, an efficient, rapid, and sensitive detection method is needed for early detection of the virus and to reduce economic losses.

Several molecular detection methods have been developed to confirm PPMV-1 infections, with reverse transcription polymerase chain reaction (RT-PCR) being the most commonly used [4, 6, 16, 19, 21]. However, RT-PCR assays are limited in terms of pathogen detection because of the need for specialized laboratory equipment and professional training, as well as their relatively time-consuming nature. As a result, RT-PCR assays are often unavailable in poorly resourced laboratories and under field conditions.

Loop-mediated isothermal amplification (LAMP) was developed by Notomi and is a rapid, simple, and sensitive technique [17] that can be carried out at a constant temperature of $60-65^{\circ} \mathrm{C}$ for $60 \mathrm{~min}$ with four to six primers that recognize six or eight specific regions of the target DNA sequence, as well as a sequence-specific DNA polymerase (Bst DNA polymerase) [18, 23]. Recently, LAMP assays have been developed for the rapid detection of important pathogens such as SARS-CoV-2 [14], dengue virus [29], JC polyomavirus (JCV) [12], and porcine senecavirus A [28]. This method is less dependent on complex instruments than RT-PCR and has been widely used to detect viruses and other pathogens $[15,29]$. However, due to the high concentrations of primers used in LAMP amplification, it is inevitable that some mismatches and primer dimers occur. This can lead to false positive results during high-speed LAMP amplification [12, 14, 28, 29].

In this study, a method combining a TaqMan probe with loop-mediated isothermal amplification (TaqMan-LAMP) was developed to prevent the false positive results associated with LAMP assays. Simultaneously, we used Bst 5.0 DNA polymerase with strong strand displacement ability, efficient DNA synthesis ability, and exonuclease activity. This method takes advantage of the rapid, highly sensitive, and constant-temperature detection that can be achieved by LAMP while mitigating the issue of false positive results. The newly developed TaqMan-LAMP is a simple and rapid method for PPMV-1 surveillance and lays a solid foundation for the wider application of LAMP technology in clinical diagnosis.

\section{Materials and methods}

\section{TaqMan-LAMP primer design}

Six PPMV-1-specific LAMP primers and a probe targeting the $F$ gene were designed based on the genomic sequence of PPMV-1 (GenBank number HM063425.1) using Primer Explorer version 5 (primerexplorer.jp/lampv5e/index.html). The primers included two outer primers (F3 and B3), two inner primers (FIP and BIP), and two loop primers (LoopF and LoopB), as well as a TaqMan probe (Fig. 1 and Table 1). Primers were synthesized by GENEWIZ (GENEWIZ, Suzhou, China), dissolved in RT-PCR-grade water (Invitrogen) to a final concentration of $100 \mu \mathrm{M}$, and stored at $-20^{\circ} \mathrm{C}$. The sequences of the primer binding sites were compared to the corresponding sites in other related viruses to identify sequence variations, insertions or deletions.

\section{RNA and DNA extraction}

Viral RNA was extracted from feces using an RNA extraction kit (QIAGEN, MD, USA). Total DNA was extracted from the DNA viruses porcine circovirus (PCV), Marek's disease virus (MDV), and egg drop syndrome virus (EDSV) using a DNA extraction kit (QIAGEN, MD, USA). The concentration and quality of the RNA and DNA was estimated by measuring the $\mathrm{OD}_{260}$ and the $\mathrm{OD}_{260} / \mathrm{OD}_{280}$ ratio using a NanoDrop Lite Spectrophotometer (Thermo Scientific, Waltham, MA, USA). RNA and DNA were stored at $-80{ }^{\circ} \mathrm{C}$ for subsequent analysis.

\section{TaqMan-LAMP assay reaction conditions}

A $20 \mu \mathrm{L}$ TaqMan-LAMP reaction mixture contained the FIP (F1c-F2) and BIP (B1c-B2) internal primers at a concentration of $16 \mu \mathrm{M}$, the $\mathrm{F} 3$ and $\mathrm{B} 3$ outer primers at a concentration of $2 \mu \mathrm{M}$, loopF and loopB at a concentration of $8 \mu \mathrm{M}$, and $8 \mu \mathrm{M}$ probe. The mixture also contained $10 \mu \mathrm{L}$ of LAMP TaqMan reagent (HaiGene, Haibin, China), $1 \mu$ of DNA or cDNA template, and nuclease free water. The reactions were performed at $65^{\circ} \mathrm{C}$ for $10 \mathrm{~s}$ and $65^{\circ} \mathrm{C}$ for $50 \mathrm{~s}$ with 25 cycles. The total time for the reaction was $25 \mathrm{~min}$.

\section{Specificity analysis of the TaqMan-LAMP assay}

The specificity of the PPMV-1 TaqMan-LAMP assay was determined using other viruses (PCV, MDV, EDSV, canine parainfluenza virus [CPIV], peste des petits ruminants virus [PPRV] vaccine strain and NDV LaSota vaccine strain), which were maintained at the Institute of Animal Sciences, Chinese Academy of Agricultural Sciences.

\section{Sensitivity and detection limit of the TaqMan-LAMP assay}

To prepare a DNA positive plasmid control, a PPMV-1 $F$ gene fragment (4550-6211) was inserted into pJET 1.2. The copy number of the DNA standard was calculated using the following formula: DNA copies $\mathrm{ml}^{-1}=$ DNA concentration $\left(\mathrm{g} \mathrm{mL}^{-1}\right) \times 6.02 \times 10^{23} /$ DNA length $\times 660$. Tenfold serial dilutions of the DNA positive plasmid standard, from $10^{-1}$ to $10^{-7}$, were used to estimate the limit of detection. 


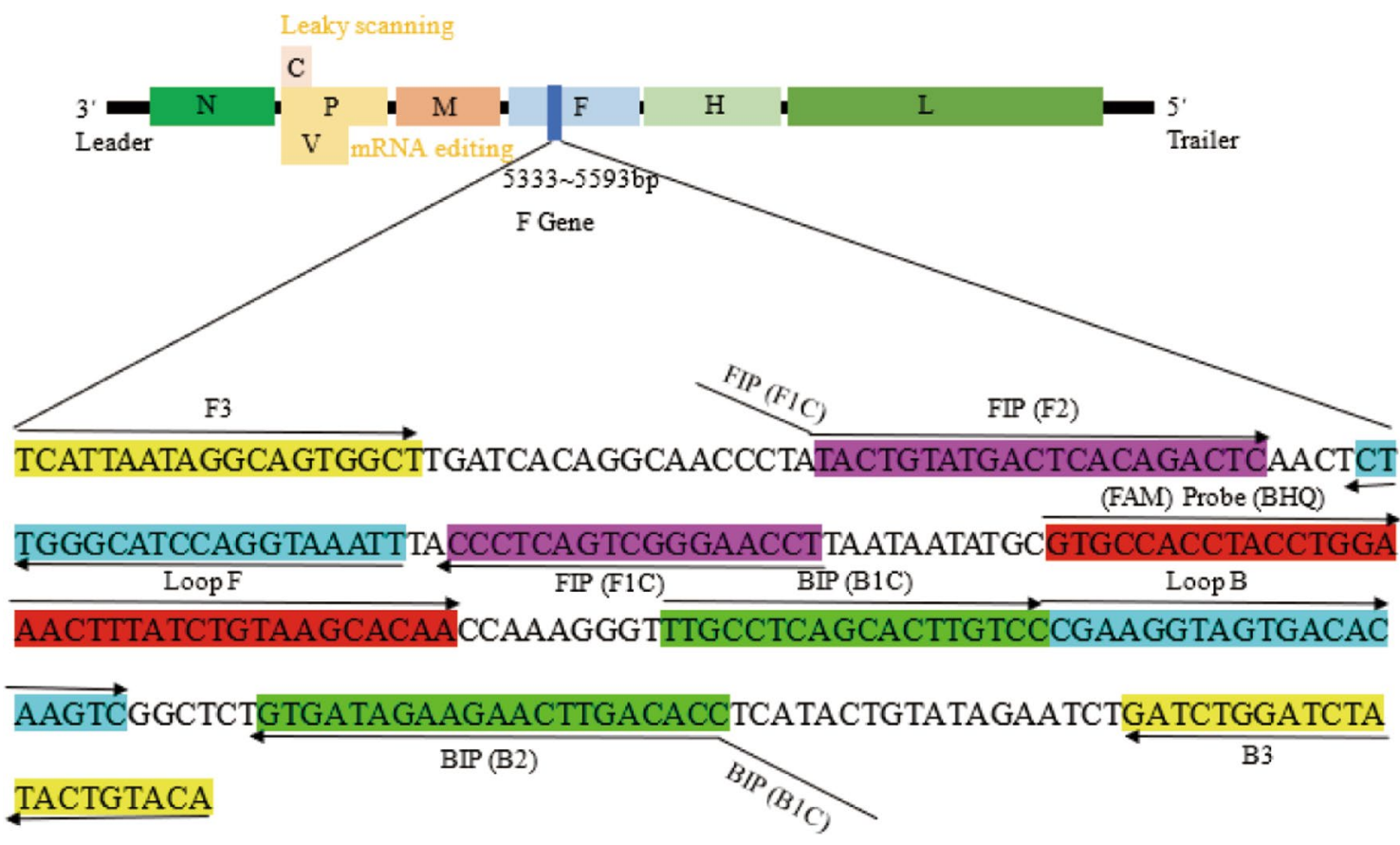

Fig. 1 Primer information. Location and partial sequences of the primer sets targeting the PPMV-1 $F$ gene. F3 and B3 are outer primers, FIP (F1c-F2) and BIP (B1c-B2) are inner primers, LoopF and LoopB are loop primers. The probe contained the fluorophore FAM (6-carboxy-fluorescein) and BHQ (Black Hole Quencher). The F1c and $\mathrm{F} 2$ sequences form the FIP primer, and $\mathrm{B} 1 \mathrm{c}$ and $\mathrm{B} 2$ form the BIP primer. The primers are depicted in different colors, with yellow used for outer primers, indigo for loop primers, magenta for FIP, green for $\mathrm{BIP}$, and red for the probe.

Table 1 Sequences of the primers and probe used in this study

\begin{tabular}{lllll}
\hline Target & Primer/probe & Sequence $\left(5^{\prime}-3^{\prime}\right)$ & Length & Position (bp) \\
\hline PPMV-1-F & F3 & TCATTAATAGGCAGTGGCT & 19 & $5333-5351$ \\
& B3 & TGTACAGTATAGATCCAGATC & 21 & $5573-5593$ \\
& FIP & AGGTTCCCGACTGAGGGTACTGTATGACTCACAGACTC & 38 & $5370-5390 / 5417-5433$ \\
& BIP & TTGCCTCAGCACTTGTCCGGTGTCAAGTTCTTCTATCAC & 39 & $5490-5507 / 5534-5554$ \\
& LoopF & AATTTACCTGGATGCCCAAG & 20 & $5395-5414$ \\
& LoopB & CGAAGGTAGTGACACAAGTC & 20 & $5508-5522$ \\
& Probe & (FAM)GTGCCACCTACCTGGAAACTTTATCTGTAAGCACAA(BHQ) & 36 & $5445-5480$ \\
\hline
\end{tabular}

\section{Clinical sample testing}

In total, 108 fecal samples from different pigeon farms were collected to evaluate the TaqMan-LAMP method. The same samples were also tested by RT-PCR as described previously [16]. RNA extraction and reverse transcription were performed using commercial kits according to the manufacturer's protocols (QIAGEN, MD, USA). Healthy pigeons were used as healthy controls, and deionized water was used as a negative control.

\section{Results}

\section{Primer design}

The LAMP primers and probes were designed for the PPMV-1 $F$ gene (GenBank accession no. MH807446.1) using LAMP designer software (Premier Biosoft International). All of the primers and probes were checked for sequence conservation by BLAST, available on the NCBI website. Then, a TaqMan-LAMP reaction with nonspecific 
amplification in the non-template control set targeting the $F$ gene was performed, which was found to have higher amplification efficiency, and was chosen for use in the development of the TaqMan-LAMP detection method. A probe with FAM as the 5' fluorophore and BHQ as the $3^{\prime}$ quencher was designed as shown in Fig. 1 and Table 1.

\section{Establishment of the TaqMan-LAMP detection method}

The reaction was performed at $65^{\circ} \mathrm{C}$ for $10 \mathrm{~s}$ and at $65^{\circ} \mathrm{C}$ for $50 \mathrm{~s}$ for 25 cycles. Reactions were performed with the newly designed primers and $1.0 \times 10^{-1} \mathrm{ng} \mathrm{L}^{-1}$ of positive plasmid control (pJET-F) or negative control (pJET). No amplification was observed for pJET, and a peak amplification time of 20 min was observed with pJET-F and PPMV-1 RNA from PPMV-1-infected pigeons (Fig. 2). After RNA was extracted from virus-infected tissues, the present method developed on the basis of the specific primer probe was used to detect different amounts of RNA. The results shown in Fig. 3, indicate that this method can detect $0.1 \mathrm{ng}$ of extracted RNA within $25 \mathrm{~min}$, but samples containing $0.01 \mathrm{ng}$ of RNA did not yield a fluorescence signal.

\section{Specificity of the PPMV-1 TaqMan-LAMP assay}

Total RNA was extracted from paramyxoviruses (CPIV, PPRV vaccine strain and NDV LaSota vaccine strain), and DNA was extracted from MDV, PCV, and EDSV. The pJEDF plasmid was used as a positive control, and distilled water was used as a negative control. Fifty nanograms of each nucleic acid was used for detection. Specificity tests showed that an amplification curve was obtained with the positive plasmid, but not with any of the other viruses (Fig. 4).

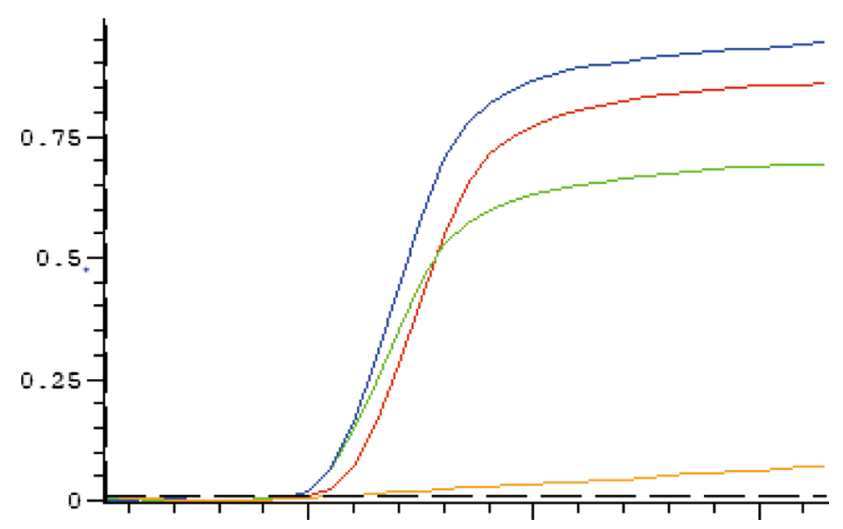

Fig. 2 Detection of PPMV-1 from PPMV-1 infected pigeons, pJET$\mathrm{F}$, and pJET by TaqMan-LAMP assay. The blue curve represents the positive plasmid control (pJET-F), the red and green curves represent RNA from infected pigeons, the orange line represents the negative control (pJET), and the green line represents the baseline threshold.

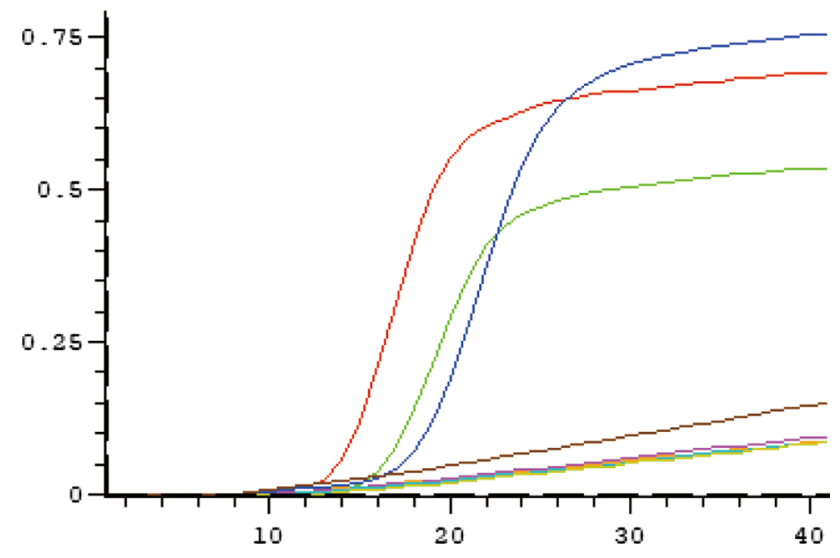

Fig. 3 Sensitivity of PPMV-1 RNA detection at different concentrations. The red, blue, and green curves represent concentrations of 10 $\mathrm{ng}, 1 \mathrm{ng}$, and $0.1 \mathrm{ng}$, respectively. The orange, magenta, turquoise, and light golden lines represent concentrations of $10^{-2} \mathrm{ng}, 10^{-3} \mathrm{ng}$, $10^{-4} \mathrm{ng}$, and $10^{-5} \mathrm{ng}$, respectively, and the sienna line represents the pJET negative control.

\section{Sensitivity of the PPMV-1 TaqMan-LAMP assay}

The PPMV-1 positive plasmid was serially diluted ten times, with seven dilutions ranging from $10^{-1}$ to $10^{-7}$, and TaqMan-LAMP amplification was performed according to the method established to confirm the validity of the procedure. Sensitivity analysis showed that, with pJET-F plasmid as the detection template, the TaqMan-LAMP method could effectively detect the standard plasmid at a concentration of 10 copies $\mu \mathrm{L}^{-1}$, and the reaction time was just $25 \mathrm{~min}$ (Fig. 5).

\section{Evaluation of the PPMV-1 TaqMan-LAMP method using clinical samples}

To evaluate the clinical application of the TaqMan-LAMP assay, it was compared with an RT-PCR assay using 108 clinical samples collected from a pigeon farm. Of those samples, 24 were positive and 84 were negative for PPMV-1 in the TaqMan-LAMP (Table 2). The concordance rate between RT-PCR and TaqMan-LAMP was $96.3 \%$.

\section{Discussion}

PPMV-1 infected pigeons can spread viruses via the oral, tracheal or cloacal route and easily infect the sentinel bird in the loft. Early detection and timely diagnosis are crucial in preventing the spread of the virus and further losses. This is especially true for PPMV-1, which is one of the most important contagious viruses affecting pigeon farms. Currently, the preferred methods of confirming the identity of the virus in infected pigeons are RT-PCR, restriction enzyme 
Fig. 4 Specificity of the TaqMan-LAMP assay. The common poultry viruses used in this assay include MDV, PCV, and EDSV. Other paramyxoviruses (CPIV, PPRV vaccine strain and NDV LaSota vaccine strain) were also tested. pJET-F and PPMV-1 RNA were used as positive controls, and pJET was used as a negative control. The red curve represents pJET-F detection, and the green curve represents PPMV-1 RNA detection.

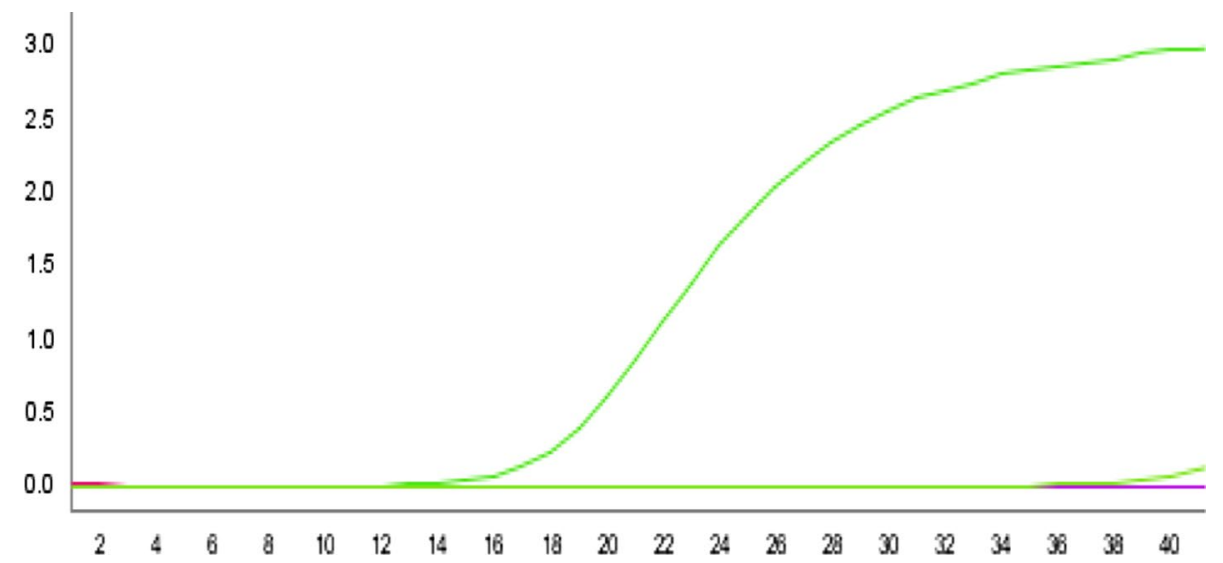

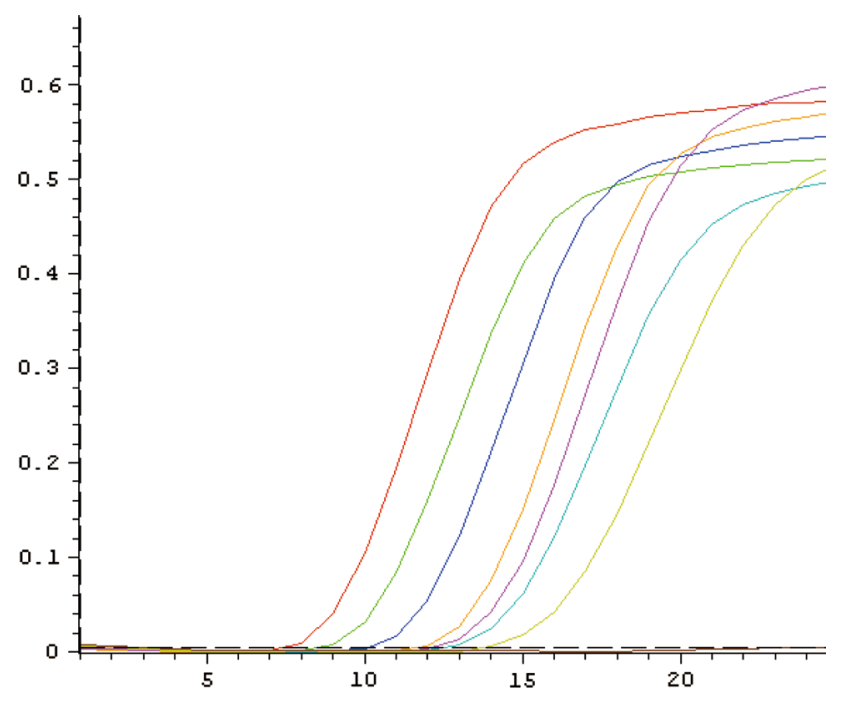

Fig. 5 Sensitivity of the TaqMan-LAMP assay determined using tenfold serial dilutions of pJET-F. Red, green, blue, orange, magenta, cyan, and light golden lines represent different dilutions containing of $10^{7}$ to 10 copies of the plasmid.

analysis, and RT-nested PCR [13, 23]. However, these methods require specialized facilities and highly trained workers and are time consuming, which renders them unsuitable for detecting pathogens under field conditions. Several studies on the development of diagnostic methods for other pathogens have shown that LAMP assays exhibit equal or even higher sensitivity when compared with qPCR assays $[8,27]$.
Probe based LAMP techniques are among the most specific and sensitive diagnostic methods available [20]. Our results indicate that the TaqMan-LAMP is a rapid, simple, accurate, and sensitive detection method that can be utilized under both laboratory and field conditions for the early diagnosis of PPMV-1 infections [20].

This is the first report of a TaqMan-LAMP assay used to detect PPMV-1. In this study, a TaqMan-LAMP diagnostic method was established for the detection of PPMV-1 in infected pigeons, using a set of six primers and a probe targeting the $F$ gene. The TaqMan-LAMP assay was optimized using RNA extracted from PPMV-1-infected pigeon faeces as a template. PPMV-1 RNA in samples was detected in as little as $25 \mathrm{~min}$, whereas PCR assays require at least $2 \mathrm{~h}$. The method was shown to be highly sensitive, with a detection limit of 10 copies per reaction, and there was no cross-reactivity with other paramyxoviruses (CPIV, PPRV, vaccine strain and NDV LaSota) or other poultry viruses (MDV, PCV, and EDSV). RT-PCR and restriction enzyme analysis are not able to distinguish PPMV-1 from other APMV-1 genotypes [16], whereas TaqMan-LAMP was found to specifically differentiate PPMV-1 from the NDV LaSota vaccine strain. The specificity of this assay derives from the use of a set of six primers and a probe targeting nine regions of the PPMV- $F$ gene. The diagnostic specificity of the TaqMan-LAMP assay was demonstrated by comparison with RT-PCR. The concordance rate between two methods was $96.3 \%$ (Table 2), indicating higher specificity of the TaqMan-LAMP method.
Table 2 Comparison of TaqMan-LAMP and RT-PCR assays

\begin{tabular}{llllll}
\hline TaqMan-LAMP & RT-PCR & Total & Positive rate (\%) & $\begin{array}{l}\text { Concordance rate } \\
(\%)\end{array}$ \\
\cline { 2 - 4 } & Positive & Negative & & & \\
\hline Positive & 20 & 4 & 24 & & \\
Negative & 0 & 84 & 84 & $22.22 \%$ & $96.3 \%$ \\
Total & 20 & 88 & 108 & & \\
Positive rate $(\%)$ & & $18.52 \%$ & & & \\
\hline
\end{tabular}


In conclusion, a sensitive and time saving TaqManLAMP assay was developed for the rapid and accurate detection of PPMV-1. This method shows excellent potential as a diagnostic tool that could be used to monitor disease in infected pigeons, to detect PPMV-1 infections when visible symptoms emerge, and to identify latent infections to prevent virus transmission and further disease outbreaks.

Author contributions RL developed the TaqMan-LAMP method and prepared the original draft; LL and KH treated samples; XR collected samples; YJ edited the manuscript; CS collected samples and performed investigations; and SC acquired funding. All authors have read and agreed to the published version of the manuscript.

Funding This work was supported by the Modern Agricultural Industry Technology System Beijing Poultry Innovation Team, grant no. BAIC-2020 and the Agricultural Science and Technology Innovation Program of China (ASTIP-IAS15).

\section{Compliance with ethical standards}

Conflict of interest The authors declare that they have no competing interests.

\section{References}

1. Aldous EW, Fuller CM, Ridgeon JH, Irvine RM, Alexander DJ, Brown IH (2014) The evolution of pigeon paramyxovirus type 1 (PPMV-1) in Great Britain: a molecular epidemiological study. Transbound Emerg Dis 61:134-139

2. Alexander DJ (2000) Newcastle disease and other avian paramyxoviruses. Rev Sci Tech Oie 19:443-462

3. Biancifiori F, Fioroni A (1983) An occurrence of Newcastle disease in pigeons: virological and serological studies on the isolates. Comp Immunol Microbiol Infect Dis 6:247-252

4. Carrasco ADT, Rodrigues JNM, Seki MC, de Moraes FE, Silva JR, Durigon EL, Pinto AA (2013) Use of reverse transcriptase polymerase chain reaction (RT-PCR) in molecular screening of Newcastle disease virus in poultry and free-living bird populations. Trop Anim Health Pro 45:569-576

5. Collins MS, Alexander DJ, Brockman S, Kemp PA, Manvell RJ (1989) Evaluation of mouse monoclonal antibodies raised against an isolate of the variant avian paramyxovirus type 1 responsible for the current panzootic in pigeons. Arch Virol 104:53-61

6. Creelan JL, McCullough SJ (2006) Detection and differentiation of pathogenicity of avian paramyxovirus serotype 1 (APMV-1) from field cases using one-step real-time RT-PCR. Dev Biologicals 126:149-157

7. de Leeuw O, Peeters B (1999) Complete nucleotide sequence of Newcastle disease virus: evidence for the existence of a new genus within the subfamily Paramyxovirinae. J Gen Virol 80(Pt 1):131-136

8. Gao W, Huang H, Zhu P, Yan X, Fan J, Jiang J, Xu J (2018) Recombinase polymerase amplification combined with lateral flow dipstick for equipment-free detection of Salmonella in shellfish. Bioprocess Biosyst Eng 41:603-611

9. Guo H, Liu X, Han Z, Shao Y, Chen J, Zhao S, Kong X, Liu S (2013) Phylogenetic analysis and comparison of eight strains of pigeon paramyxovirus type 1 (PPMV-1) isolated in China between 2010 and 2012. Arch Virol 158:1121-1131
10. Guo H, Liu X, Xu Y, Han Z, Shao Y, Kong X, Liu S (2014) A comparative study of pigeons and chickens experimentally infected with PPMV-1 to determine antigenic relationships between PPMV-1 and NDV strains. Vet Microbiol 168:88-97

11. He Y, Lu B, Dimitrov KM, Liang J, Chen Z, Zhao W, Qin Y, Duan Q, Zhou Y, Liu L, Li B, Yu L, Duan Z, Liu Q (2020) Complete genome sequencing, molecular epidemiological, and pathogenicity analysis of pigeon paramyxoviruses type 1 isolated in Guangxi, China during 2012-2018. Viruses 12:366

12. Kinoshita H, Nakamichi K, Lim CK, Takayama-Ito M, Wang L, Iizuka I, Kurane I, Saijo M (2018) A loop-mediated isothermal amplification assay for the detection and quantification of JC polyomavirus in cerebrospinal fluid: a diagnostic and clinical management tool and technique for progressive multifocal leukoencephalopathy. Virol J 15:136

13. Liu H, Wang Z, Son C, Wang Y, Yu B, Zheng D, Sun C, Wu Y (2006) Characterization of pigeon-origin Newcastle disease virus isolated in China. Avian Dis 50:636-640

14. Lu R, Wu X, Wan Z, Li Y, Jin X, Zhang C (2020) A novel reverse transcription loop-mediated isothermal amplification method for rapid detection of SARS-CoV-2. Int J Mol Sci 21:344-347

15. Lu RF, Wu XM, Wan ZZ, Li YX, Jin X, Zhang CY (2020) A novel reverse transcription loop-mediated isothermal amplification method for rapid detection of SARS-CoV-2. Int J Mol Sci 21:2826

16. Naveen KA, Singh SD, Kataria JM, Barathidasan R, Dhama K (2013) Detection and differentiation of pigeon paramyxovirus serotype-1 (PPMV-1) isolates by RT-PCR and restriction enzyme analysis. Trop Anim Health Prod 45:1231-1236

17. Notomi T, Okayama H, Masubuchi H, Yonekawa T, Watanabe K, Amino N, Hase T (2000) Loop-mediated isothermal amplification of DNA. Nucleic Acids Res 28:63

18. Parida M, Sannarangaiah S, Dash PK, Rao PVL, Morita K (2008) Loop mediated isothermal amplification (LAMP): a new generation of innovative gene amplification technique; perspectives in clinical diagnosis of infectious diseases. Rev Med Virol 18:407-421

19. Pham HM, Konnai S, Usui T, Chang KS, Murata S, Mase M, Ohashi K, Onuma M (2005) Rapid detection and differentiation of Newcastle disease virus by real-time PCR with melting-curve analysis. Adv Virol 150:2429-2438

20. Soltani Tehrani B, Mirzajani E, Fallahi S, Manouchehri Naeini K, Mahmoudi MR, Safari Kavishahi M, Eskandari V, Zebardast N (2020) Challenging TaqMan probe-based real-time PCR and loop-mediated isothermal amplification (LAMP): the two sensitive molecular techniques for the detection of toxoplasmosis, a potentially dangerous opportunistic infection in immunocompromised patients. Arch Microbiol 202:1881-1888

21. Steyer AF, Rojs OZ, Krapez U, Slavec B, Barlic-Maganja D (2010) A diagnostic method based on MGB probes for rapid detection and simultaneous differentiation between virulent and vaccine strains of avian paramyxovirus type 1 . J Virol Methods 166:28-36

22. Tian Y, Xue R, Yang W, Li Y, Xue J, Zhang G (2020) Characterization of ten paramyxovirus type 1 viruses isolated from pigeons in China during 1996-2019. Vet Microbiol 244:108661

23. Tomita N, Mori Y, Kanda H, Notomi T (2008) Loop-mediated isothermal amplification (LAMP) of gene sequences and simple visual detection of products. Nat Protoc 3:877-882

24. Toro H, Hoerr FJ, Farmer K, Dykstra CC, Roberts SR, Perdue M (2005) Pigeon paramyxovirus: association with common avian pathogens in chickens and serologic survey in wild birds. Avian Dis 49:92-98

25. Ujvari D (2006) Complete nucleotide sequence of IT-227/82, an avian paramyxovirus type-1 strain of pigeons (Columba livia). Virus Genes 32:49-57 
26. Wang J, Liu H, Liu W, Zheng D, Zhao Y, Li Y, Wang Y, Ge S, Lv Y, Zuo Y, Yu S, Wang Z (2015) Genomic characterizations of six pigeon paramyxovirus type 1 viruses isolated from live bird markets in China during 2011 to 2013. PLoS ONE 10:e0124261

27. Wu X, Song Z, Zhai X, Zuo L, Mei X, Xiang R, Kang Z, Zhou L, Wang $H$ (2019) Simultaneous and visual detection of infectious bronchitis virus and Newcastle disease virus by multiple LAMP and lateral flow dipstick. Poult Sci 98:5401-5411

28. Zeng F, Cong F, Liu X, Lian Y, Wu M, Xiao L, Yuan W, Huang R, Ma J, Guo P, Luo M (2018) Development of a real time loopmediated isothermal amplification method for detection of Senecavirus A. J Virol Methods 261:98-103
29. Zhou Y, Wan Z, Yang S, Li Y, Li M, Wang B, Hu Y, Xia X, Jin X, Yu N, Zhang C (2019) A Mismatch-tolerant reverse transcription loop-mediated isothermal amplification method and its application on simultaneous detection of all four serotype of dengue viruses. Front Microbiol 10:1056

Publisher's Note Springer Nature remains neutral with regard to jurisdictional claims in published maps and institutional affiliations. 